\title{
Diffusion in a stochastic magnetic field in ASDEX Upgrade
}

\author{
O. Dumbrajs ${ }^{1,2}$, V. Igochine ${ }^{3}$, H. Zohm ${ }^{3}$ and ASDEX Upgrade Team ${ }^{3}$ \\ ${ }^{1}$ Institute of Solid State Physics, Association Euratom-University of Latvia, Kengaraga Street 8, LV-1063, Riga, Latvia \\ ${ }^{2}$ Helsinki University of Technology, Association Euratom-Tekes, P.O.Box 2200, FIN-02015 HUT, Finland \\ ${ }^{3}$ MPI für Plasmaphysik, Euratom-Association, D-85748 Garching, Germany
}

\begin{abstract}
Heat diffusion coefficients in a stochastic magnetic field are determined in the case of frequently interrupted regime of neoclassical tearing modes and of incomplete sawtooth reconnection in ASDEX Upgrade tokamak. Here the experimentally measured perturbations and profiles are used and the mapping technique is applied. With the derived diffusion coefficients the nonstationary diffusion equation is solved, making it possible to study time evolution of fast MHD phenomena in ASDEX Upgrade. The proposed phenomenological approach relies heavily on experimental information and requires very moderate computing resources.
\end{abstract}

\section{Introduction}

Fast stochastic events in ASDEX Upgrade are relatively unexplored [1]. Stochasticity of magnetic field lines, introduced by helical perturbations due to MHD instabilities, is believed to play a role in fast reconnection phenomena in magnetized plasmas. These fast reconnection phenomena are often accompanied by sharp drops in the temperature, also referred to as 'crash'. The chaotic field lines in the stochastic region contribute to the radial energy and particle transport. Such a transport can be characterized by diffusion coefficients. In $[2,3]$ it was found that fast reconnection observed during amplitude drops of the neoclassical tearing mode instability in the frequently interrupted regime can be related to stochastization. Recently [4] it was also demonstrated that amplitudes of the perturbations are sufficient to create a large stochastic region during sawtooth reconnection. In these papers stationary situations were considered with stochastization caused by perturbations which were deduced from the 
experiment. In the present paper we focus our attention on the time evolution of the system, in particular on the heat diffusivity.

It is clear that the full dynamics of instabilities cannot be described within the framework of the field line tracing approach. Here full 3D non-linear MHD calculations with parallel and perpendicular transport are needed. Unfortunately, such 3D non-linear MHD calculations are not possible with experimentally relevant plasma parameters. With present day computers only moderate magnetic Reynolds numbers $\left(10^{6}\right)$ can be processed which are some orders of magnitude smaller than experimental values $\left(10^{9}-10^{10}\right)$. Moreover the spatial and mode resolutions are restricted. At the same time, the field line mapping technique provides correct representation of the field line stochasticity (all toroidal effects are taken into account) and it can be carried out on a personal computer. In this manner diffusion coefficients which correspond to experimental plasma perturbations can be obtained under the assumption that the electrons experience many turns around the torus on the time scale on which the MHD mode varies. This allows us to investigate dynamic behaviour of the temperature profile and to understand the limits and driving forces of stochastic processes in tokamaks where of course the temporal evolution of the MHD instability is not modelled self-consistently, but taken from experimental data.

Before going into details, it is important to emphasize that in our approach only the transport parallel to the magnetic field is taken into account. This assumption is valid only for fast evolution of the system which is much faster than usual radial transport time scales in plasma. It is a satisfactory model already for slightly stochastic cases and is a good model for all cases considered below.

The paper is organised as follows. In section 2, we discuss the derivation of diffusion coefficients by means of the mapping technique. In section 3, we solve numerically the nonstationary diffusion equation. In section 4 we summarize the main results.

\section{Diffusion coefficients}

In typical Hamiltonian systems like magnetic field lines in fusion plasmas the zone of chaotic motion is not uniform, especially the stochastic layer near the separatrix. 
It consists of KAM-stability islands embedded in a so-called stochastic sea. The structure of the stochastic layer is determined by mutual positions and sizes of KAM-islands. Existence of these islands leads to the deviation of chaotic motion from the normal diffusion process described by the Gaussian random walk approximation. Departure of the statistics of chaotic motion from the Gaussian one is called anomalous diffusion. ${ }^{\text {a }}$ The anomalous diffusion along the radial coordinate $r$ is characterized by a nonlinear dependence of the second moment displacement $\sigma^{2}(l)$ on the distance $l$ along the field line

$$
\sigma^{2}(l)=\frac{1}{N} \sum_{i=1}^{N}\left[\frac{r_{i}(l)}{a}-\frac{r}{a}\right]^{2}=2 D l^{\gamma}
$$

Here $N$ is the number of initial conditions at $l=0$ taken on a certain magnetic surface $r / a$, where $a$ is the minor radius of the tokamak, and $D$ determines a diffusion coefficient. For a normal Gaussian process the exponent $\gamma=1$. The case $\gamma>1$ is known as enhanced (superdiffusive) transport, while the case $\gamma<1$ describes a reduced (subdiffusive) transport. It should be emphasized that equation (1) defines the diffusion coefficient in an infinite domain. Because the mapping models produce stochastic zones of a finite size, one can not use equation. (1). However, one can introduce [5,6] the socalled local diffusion coefficient

$$
D_{F L}=\frac{\sigma^{2}(l)}{2 l}
$$

at the length $l$, where the growth of $\sigma^{2}(l)$ is linear. It gives a quantitative measure of field line diffusion near the given magnetic surface of radius $r / a$. The distance $l$ along a field line may be related to the number of steps $k$ in the mapping: $l=\pi R k / 2$ where $R$ is the major radius of the tokamak.

\subsection{Frequently interrupted regime of the neoclassical tearing modes}

\footnotetext{
${ }^{a}$ We note that here the term ,anomalous diffusion“ does not refer to the turbulent transport in the plasma.
} 
Using the mapping technique which is described in detail in [3] we first recalculated the Poincare section of the field lines corresponding to the interaction of the $(3,2)$ and $(4,3)$ modes for an ASDEX Upgrade experimental situation described in [3]. This plot is shown in figure 1 (compare with figure 4 in [3], note that $\psi=r^{2} / 2$ ). The plot was obtained with 165 initial values of the radial coordinate evenly distributed in the interval $0 \leq \frac{r}{a} \leq 1.0$. Here $\theta_{0}=0$ in all cases and 1000 iterations were used which corresponds to 1000 toroidal circuits because the largest mapping step $2 \pi$ was used. The radial dependence of the local diffusion coefficient corresponding to this Poincare plot is shown in figure 2 . Here averaging over a set of $N=360$ initial values of poloidal angle was performed: $0 \leq \theta_{0}<2 \pi$.

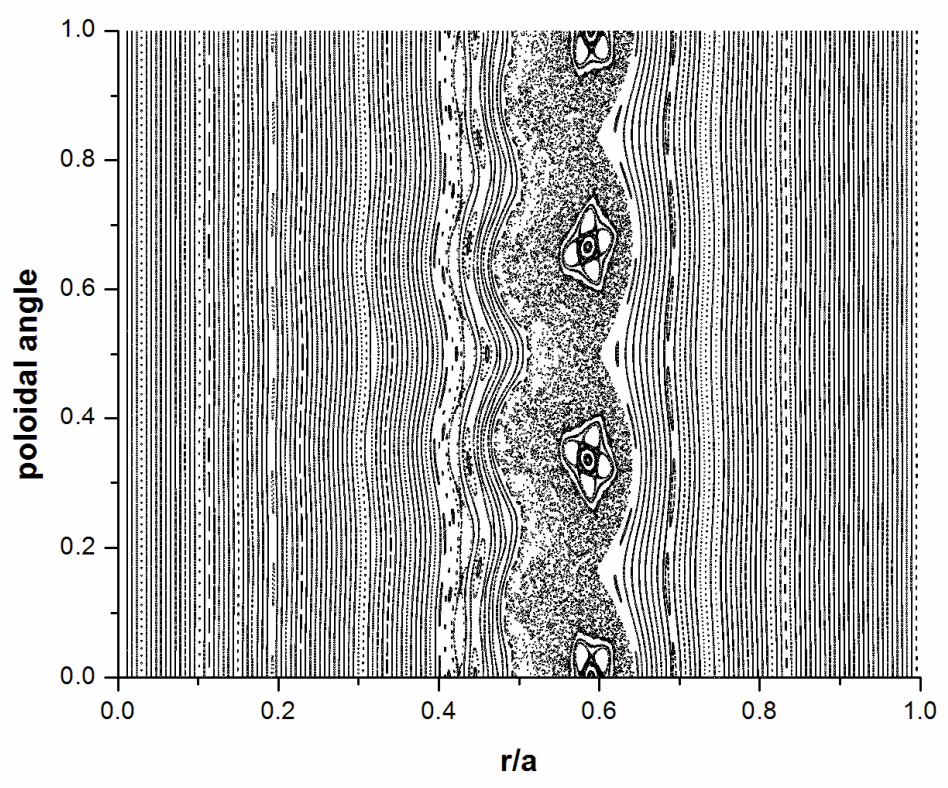

Figure 1. Poincare plot corresponding to interaction of the $(3,2)$ and $(4,3)$ modes during the FIR-NTM regime. 


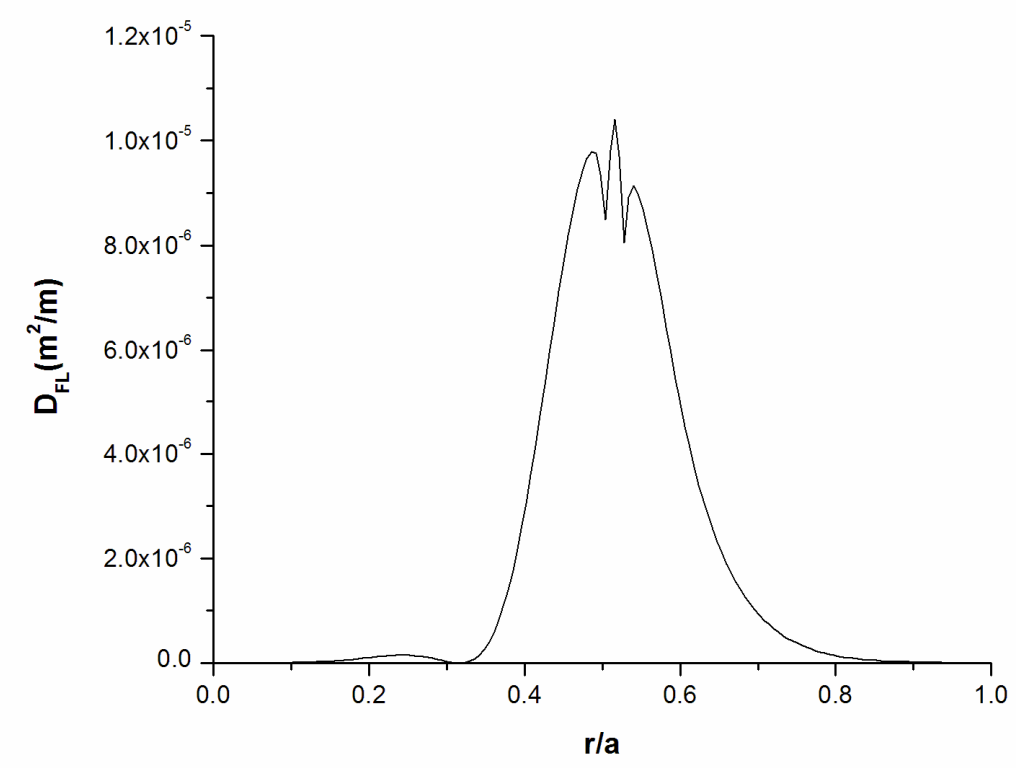

Figure 2. Local diffusion coefficient corresponding to Poincare plot shown in figure 1.

It is evident that the local diffusion coefficient grows with $r / a$ until approximately middle of the stochastic zone, then monotonically decreases over the $(3,2)$ islands located at $r / a=0.592$ to the laminar zone. It is interesting to note that the maximal value of the coefficient is of the order of $10^{-5}\left[\mathrm{~m}^{2} / \mathrm{m}\right]$ which is of the same order of magnitude as found in the studies of ergodic divertor magnetic fields in toroidal systems [5].

\subsection{Sawtooth crash}

In figures 3 and 4 we show Poincare plots corresponding to the $(1,1),(2,2)$ and $(3,3)$ mode interaction during the sawtooth crash (compare with figure 10 in [4]). 


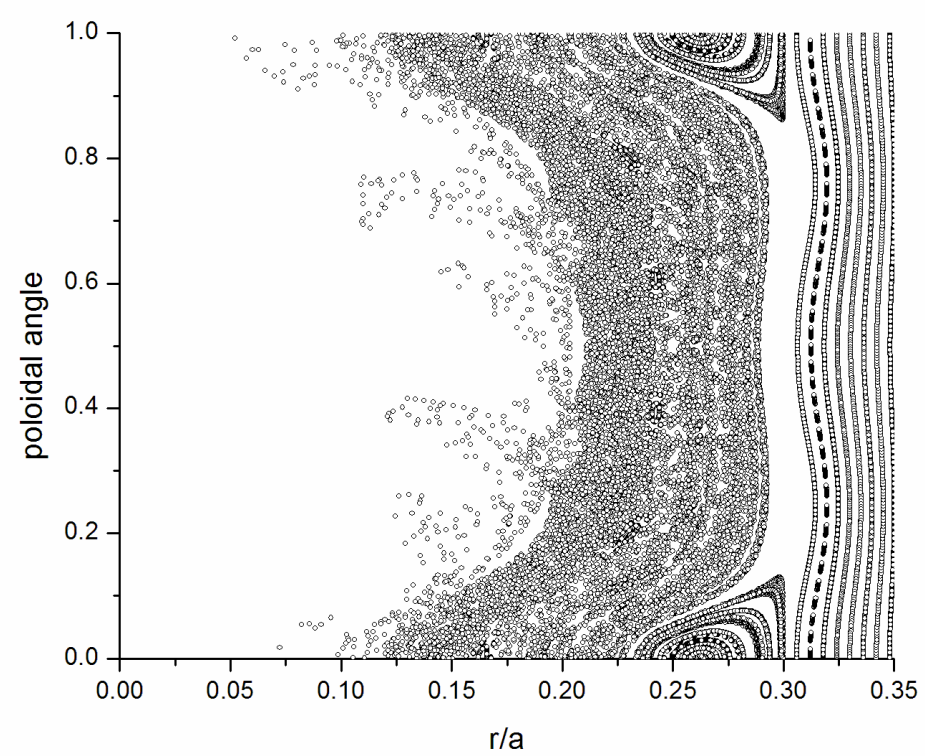

Figure 3. Poincare plot corresponding to perturbations $(1,1)+(2,2)+(3,3)$. Here the $(1,1)$ amplitude is $6 \mathrm{~cm}$.

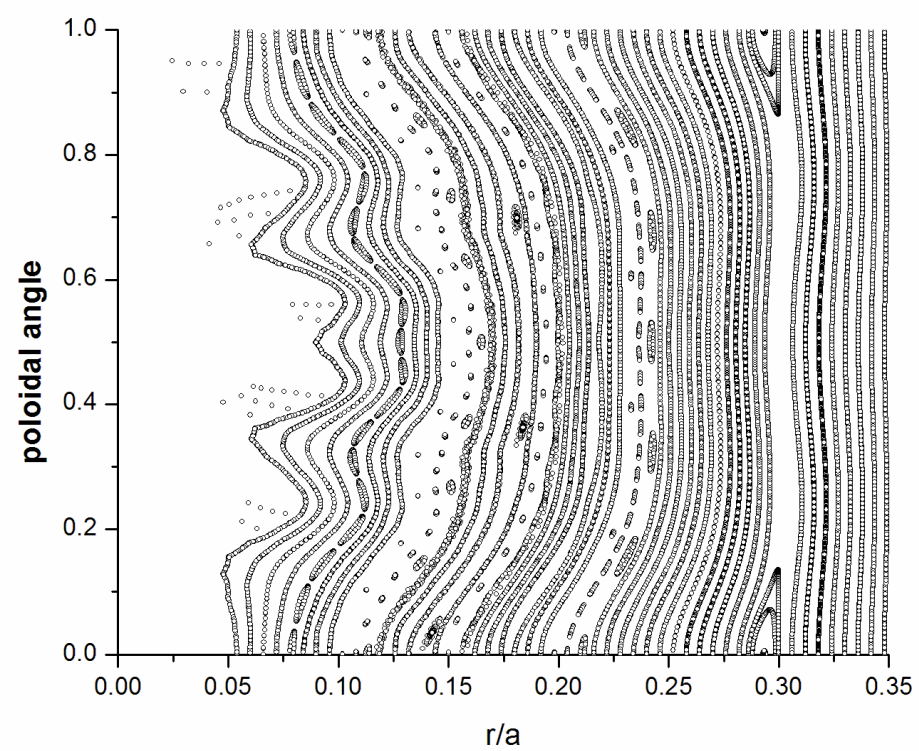

Figure 4. Poincare plot corresponding to perturbations $(1,1)+(2,2)+(3,3)$. Here the $(1,1)$ amplitude is $3 \mathrm{~cm}$. 
The radial dependence of the local diffusion coefficient corresponding to these Poincare plots is shown in figures 5 and 6.

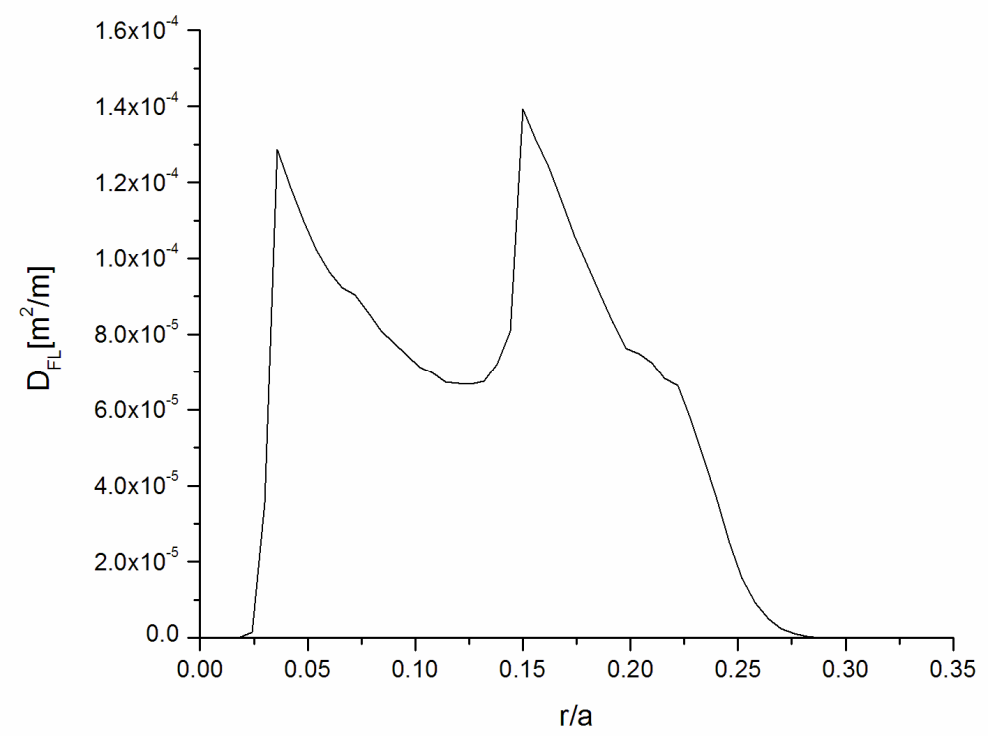

Figure 5. Local diffusion coefficient corresponding to Poincare plot shown in figure 3.

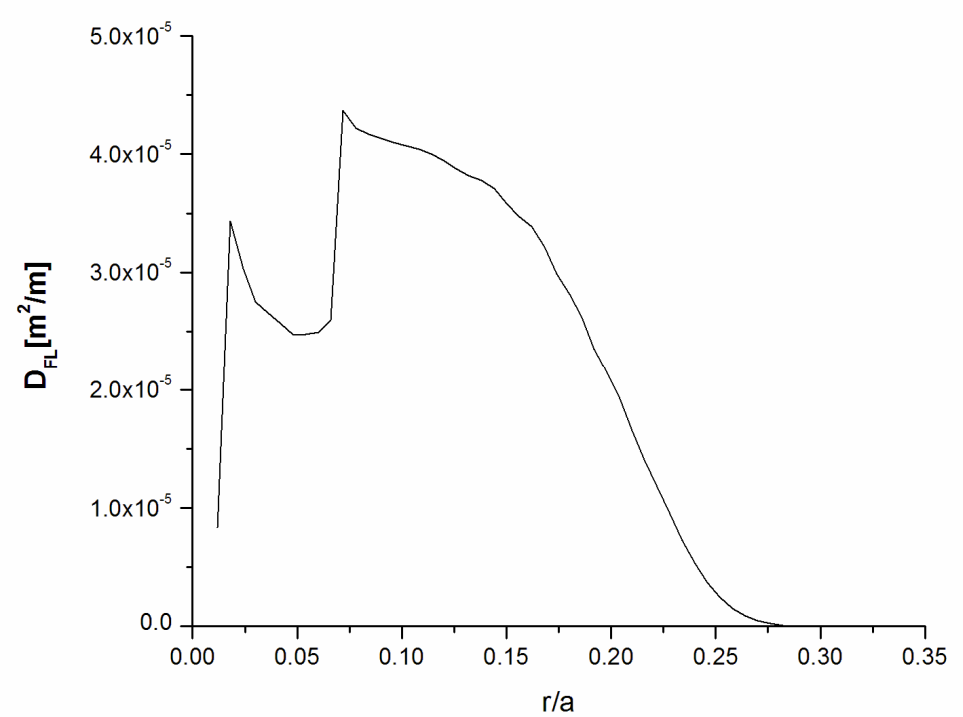

Figure 6. Local diffusion coefficient corresponding to Poincare plot shown in figure 4. 
It is evident that both the Poincare plots and the local diffusion coefficient radial dependencies in the case of FIR-NTM are quite different compared to a sawtooth crash. In the former case stochastization is caused by a simple overlapping of the $(3,2)$ and $(4,3)$ modes, while in the later case stochastization strongly depends on a subtle interplay of the low-order rational surfaces [4] resulting in tooth-like structures in Poincare plots (figures 3 and 4) and corresponding spikes in diffusion coefficient plots (figures 5 and $6)$.

The derived diffusion coefficients can be transformed into the electron thermal diffusivity $\chi_{e}$ by means of the expression [7]:

$$
\chi_{e}=\frac{3}{\sqrt{\pi}} v_{e} D_{F L}
$$

Assuming that the electron temperature $T_{e}=3 \mathrm{keV}$, we find that the electron thermal velocity $v_{e}=1.33 \cdot 10^{7} T_{e}^{1 / 2}=2.3 \cdot 10^{7} \mathrm{~m} / \mathrm{s}$. This gives the relation $\chi_{e}=3.9 \cdot 10^{7} D_{F L}$ $\left[\mathrm{m}^{2} / \mathrm{s}\right]$, leading to $\chi_{e}$ of the order of $10^{2}-10^{3} \mathrm{~m}^{2} / \mathrm{s}$, which is much bigger than the usual turbulent perpendicular transport, but agrees well with the electron heat transport measured in a stochastic magnetic field in the Madison Symmetric Torus [8]. ${ }^{\mathrm{b}}$

\section{Numerical solution of the nonstationary diffusion equation.}

In order to study different time scales, we solve numerically the nonstationary diffusion equation:

$$
\frac{\partial T}{\partial t}=\frac{\partial}{\partial r}\left(\chi(r, t) \frac{\partial T}{\partial r}\right)
$$

Equation (4) has to be supplemented by the initial condition

$$
T\left(\frac{r}{a}, 0\right)=f\left(\frac{r}{a}\right)
$$

\footnotetext{
${ }^{\mathrm{b}}$ In principle the electron thermal diffusivity is somewhat smaller due to the temperature change which accompanies diffusion. However estimates show [9] that the correction is smaller than $10 \%$.
} 
The experimental temperature profile at ASDEX Upgrade before the FIR event (\#11681, $\mathrm{t}=2.98 \mathrm{~s}$ ) can be parametrized as follows:

$$
f(r / a)=4.5 \cdot\left(1-\left(\frac{r}{a}\right)^{2}\right)^{2}
$$

and before the sawtooth crash (\#20975, $\mathrm{t}=4.13 \mathrm{~s})$ as follows:

$$
f(r / a)=2.46 \cdot\left(1-\left(\frac{r}{a}\right)^{2}\right)^{1.3}
$$

\subsection{Frequently interrupted regime of the neoclassical tearing modes}

In the case of FIR we impose the Dirichlet boundary conditions at both sides

$$
T(0, t)=f(0), \quad T(1, t)=f(1)
$$

The results of the calculations with local diffusion coefficients shown in figure 2 are presented in figure 7.

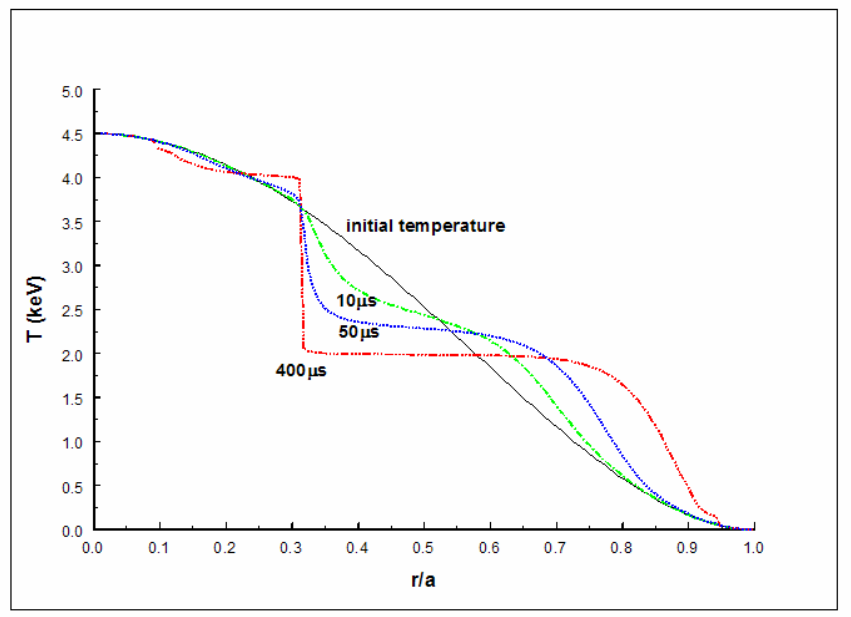

Figure 7. Temporal temperature profile evolution in the case of interaction of $(3,2)$ and $(4,3)$ modes during the FIR-NTM regime. 
The structure of the curves seen in figure 7 can be explained by the fact that the diffusion coefficient used in the calculations (figure 2) corresponds to the parallel diffusion which is negligible outside the stochastic region (figure 1). The perpendicular component governing diffusion in laminar regions is not taken into account in our model. As a result for $r / a \leq 0.3$ and $r / a \geq 0.95$ the temperature profile does not depend on time. $^{\mathrm{c}}$

Experimental measurements show that the FIR drop appears on the time scale of a few hundreds of $\mu s$ [10]. During this time the temperature flattening region of about 8$10 \mathrm{~cm}$ is observed. This region includes $(3,2)$ and $(4,3)$ modes and corresponds to the time moment when these modes are coupled via the $(1,1)$ mode. The stochastization appears only during this very short time period when all the modes are coupled. Without coupling the modes have different frequencies, the corresponding perturbations are screened and no stochasticity is observed even if the amplitudes of the modes are sufficiently high. One can see in figure 7 that already after $10 \mu s$ the flattening region extends to the experimentally measured values $8-10 \mathrm{~cm}$ (from 0.4 to about 0.6 in r/a). For subsequent time moments the flattening is much larger and it reaches saturation after about $30-50 \mu \mathrm{s}$. Later changes are very small (figure $7, \mathrm{t}=400 \mu \mathrm{s}$ ). Thus, the resulting time needed for the temperature flattening is much smaller than the experimentally observed (hundreds of $\mu s$ ). This means that the diffusion is not the limiting factor which determines the time scale of the temperature drop. Instead the main constraint may be imposed by the growth rate of the $(1,1)$ mode which couples the $(3,2)$ and $(4,3)$ modes. The temperature can be redistributed during the very short time when the coupling is complete.

\footnotetext{
${ }^{\mathrm{c}}$ The diffusion coefficient is not zero between $r / a \geq 0.85$ and $r / a \leq 0.95$. This cannot be seen in figure 2 due to the scale.
} 


\subsection{Sawtooth crash}

The sawtooth crash is a very fast and complex event. This is the reason why most of the papers focus on the pre-crash and/or post-crash phases of the event. The crash itself is local not only in the poloidal direction but also in the toroidal direction as demonstrated in recent 2D temperature measurements [11]. This poses great difficulties for reconstruction of the crash, because the detection is possible typically in one poloidal position and the rotation symmetry can not be applied since the time of the crash is a few times shorter than the rotation period of the mode.

We try to reconstruct the amplitude drop during the crash phase. For this purpose we process all the experimental information about the crash using the heat diffusion equation. The assumed dynamics of the crash event is shown in figure 8 . In the first phase, the $(1,1)$ mode grows alone. Higher harmonics appear close to the crash phase and destroy the symmetrical $m=1$ structure of the mode without causing stochastization for which reconnection is needed to destroy the $q=1$ surface. This occurs at the top of the mode amplitude leading to stochastization of the internal region and to decrease of the central temperature. As the starting time of the crash event we assume the time moment at which heat begins to flow intensively through the X-point of the $(1,1)$ island outside the $q=1$ resonant surface which corresponds to the drop of the plasma temperature and can be deduced from SXR measurements. The crash is regarded to be fait accompli at the time moment when the temperature becomes almost flat in the centre. Here the size of the $(1,1)$ reduces to a stationary post-crash value and the heat channel at the X-point closes again which is shown as the final phase in figure 8. The 2D ECE measurements [11] show deformation of the internal region inside the $q=1$ surface just before the crash which can be interpreted as an influence of the higher harmonics on the $(1,1)$ mode. 
Subsequently the deformed mode several times tries to break the $q=1$ surface until a fast crash occurs. ${ }^{\mathrm{d}}$

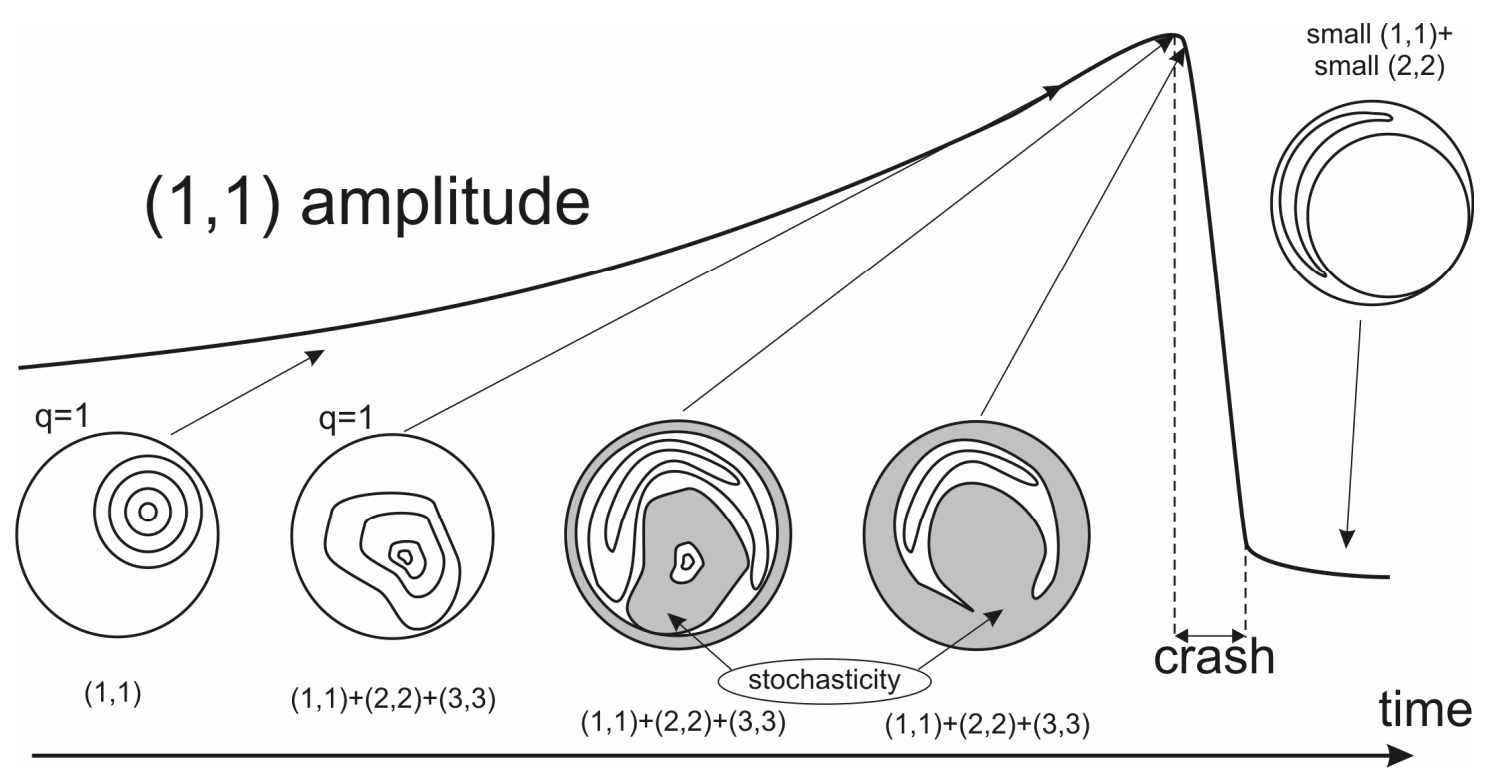

Figure 8. Artist's view of the temporal evolution of a sawtooth crash. Close to the crash phase higher harmonics grow and deform the surface, but only after reconnection stochastization becomes possible which leads to the crash.

It should be emphasized that in what follows only the region inside the $q=1$ surface has been considered, because just this region is stochastic due to perturbations (figures 3, 4 and 8) and here a strong perpendicular heat flow takes place. After the crash heat is redistributed poloidaly around the $q=1$ resonant surface, as observed also in [11]. Beyond this surface the magnetic field remains laminar all the time.

We impose the Neumann boundary condition at the plasma interior:

$$
\left.\frac{\partial T}{\partial r}\right|_{r=0}=0
$$

and the Dirichlet boundary condition

$$
T(0.30, t)=f(0.30)
$$

\footnotetext{
${ }^{\mathrm{d}}$ Possibly the described phenomenon can be interpreted as a manifestation of a hysteresis. This will be a subject of further studies.
} 
at the position of the $q=1 \operatorname{surface}\left(\frac{r}{a}=0.30\right)$ e . Here we introduce the averaged diffusion coefficient defined as follows:

$$
\bar{D}_{F L}=\frac{1}{0.35} \int_{0}^{0.30} D_{F L}\left(\frac{r}{a}\right) \frac{d r}{a}
$$

The corresponding averaged electron thermal diffusivity $\chi_{\text {aver }}=3.9 \cdot 10^{7} \bar{D}_{F L}$ as a function of the displacement of the $(1,1)$ mode is shown in figure 9 .

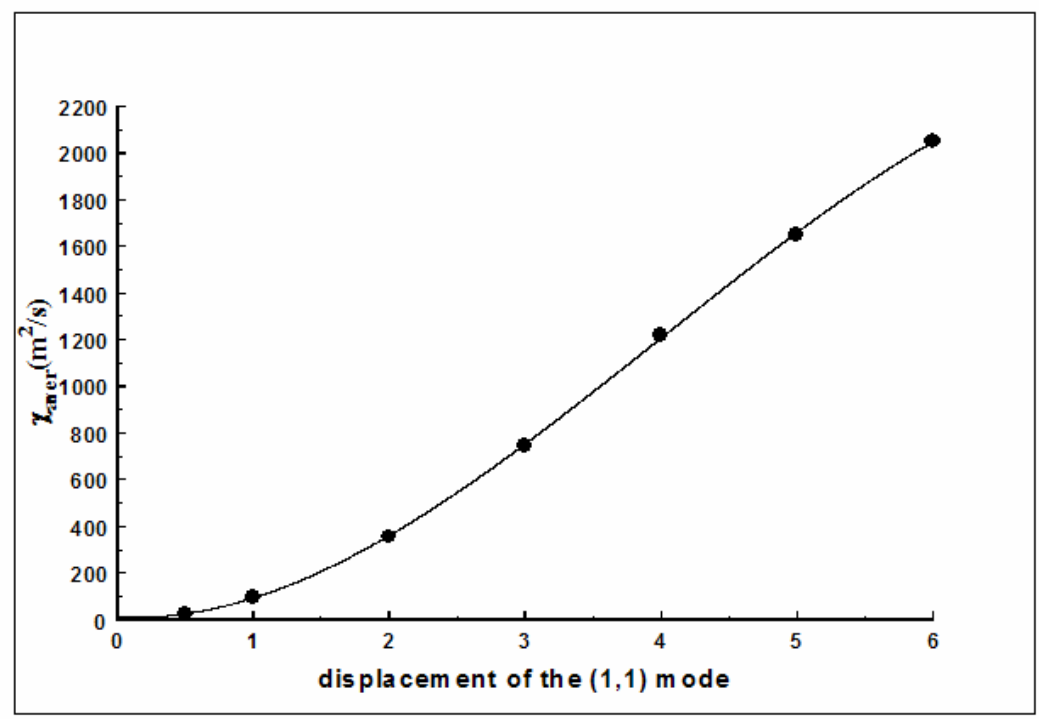

Figure 9. Dots are averaged values of the electron thermal diffusivity calculated for the displacement $\xi$ of the $(1,1)$ mode equal to $0.5,1,2,3,4,5$, and $6 \mathrm{~cm}$. The curve corresponds to the fit (12).

The dots shown in figure 9 can be parametrized by the polynomial

$$
\chi_{\text {aver }}=-10.53 \xi^{3}+125.69 \xi^{2}-35.86 \xi+12.77
$$

The $(2,2)$ and $(3,3)$ harmonics are also present $[4]$ and we scale them with the same law as the primary $(1,1)$ mode. According to experiments we assume that during the sawtooth crash $\left(\tau_{\text {crash }} \approx 40 \mu s\right)$ the displacement of the $(1,1)$ mode decays as follows:

\footnotetext{
${ }^{\mathrm{e}}$ It should be noted that because of the radial symmetry we could use also the Dirichlet boundary condition $T(-0.30, t)=T(+0.30, t)$ which mathematically is equivalent to condition (9).
} 


$$
\xi(t)=\xi_{\max }\left[1-\frac{5}{6}\left(\frac{t}{\tau_{\text {crash }}}\right)^{\alpha}\right]
$$

where $\xi_{\max }=6 \mathrm{~cm}$ and $\alpha$ characterizes the slope of the decay. Here it is assumed that the displacement of the $(1,1)$ mode during the Sawtooth crash decreases from $6 \mathrm{~cm}$ to $1 \mathrm{~cm}$ which is typical for the incomplete reconnection in ASDEX Upgrade tokamak. This parametrization can be compared with the parametrization representing the ideal MHD time scale:

$$
\xi(t)=\xi_{\max } \exp \left(-\frac{t}{\tau_{\text {Alfven }}}\right)
$$

where $\tau_{\text {Alfven }}=2 \pi R / v_{\text {Alfven }}$ is the Alfven time and $v_{\text {Alfven }}=2.18 \cdot 10^{16} \frac{B_{\phi}}{\sqrt{n}} \approx 6.5 \frac{\mathrm{m}}{\mu \mathrm{s}}$ is the Alfven velocity, or with the Kadomtsev model:

$$
\xi(t)=\xi_{\max } \exp \left(-\frac{t}{\tau_{\text {Kadomtsev }}}\right)
$$

where $\tau_{\text {Kadomtsev }}=\sqrt{\tau_{\text {res }} \cdot \tau_{\text {Alfven }}}$ is the Kadomtsev time and $\tau_{\text {res }}=\mu_{0} r_{q=1}^{2} / \eta$ is the resistive diffusion time. In ASDEX Upgrade $r_{q=1}=0.15 \mathrm{~m}$ and the resistivity $\eta=2 \cdot 10^{-9} \mathrm{ohm} \cdot \mathrm{m}$. 


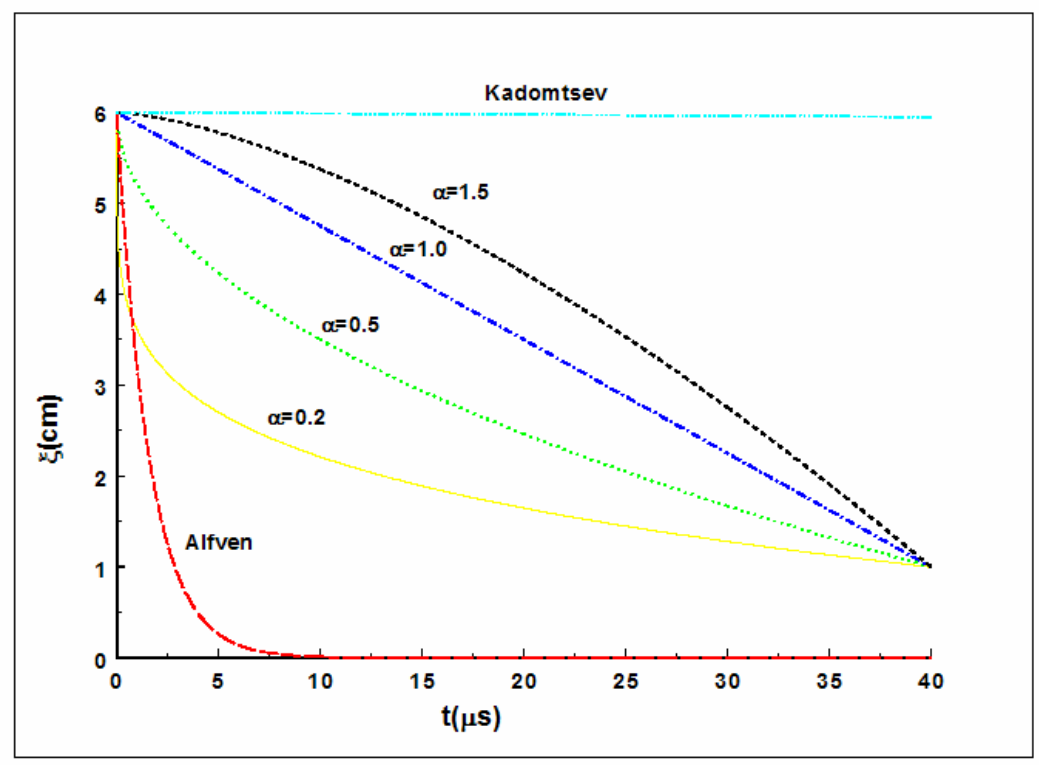

Figure 10. Temporal dependence of the displacement of the $(1,1)$ mode for different values of the parameter $\alpha$ in equation (13).

Now using equations (12), (13), (14) and (15), we obtain a time-dependent parameterization for the averaged electron thermal diffusivity. The resulting curves are shown in figure 11.

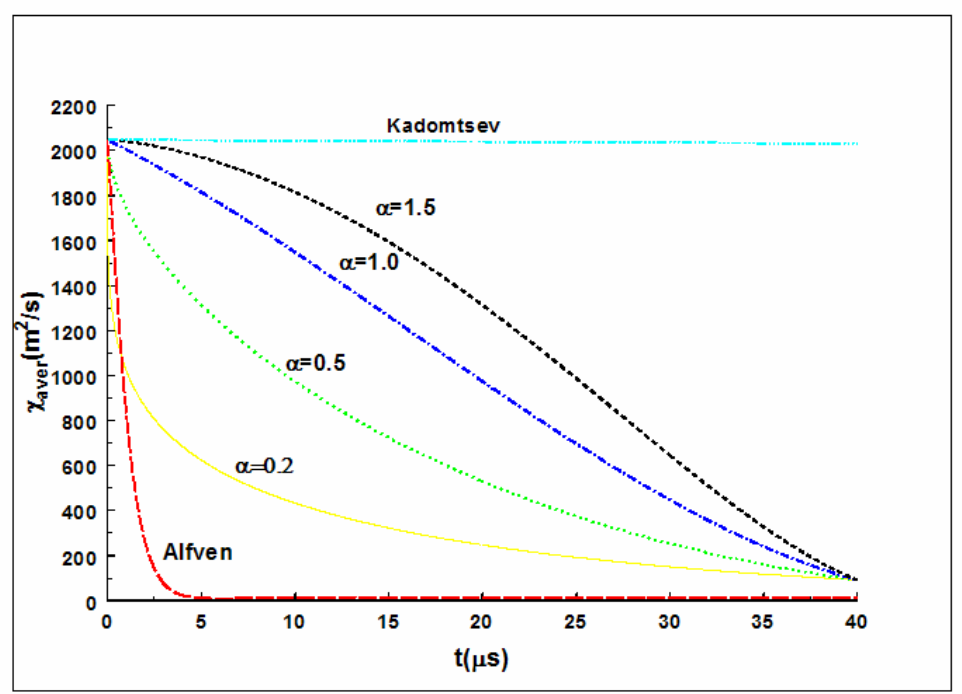

Figure 11. Temporal dependence of the averaged value of the electron thermal diffusivity for different values of the parameter $\alpha$ in equation (13). 
Solving equation (4) with $\chi_{\text {aver }}$ shown in figure 11 , we obtain temporal dependence of the temperature. In figure 12 we show the evolution of the temperature at the plasma center and in figure 13 the temperature profile after $40 \mu s$.

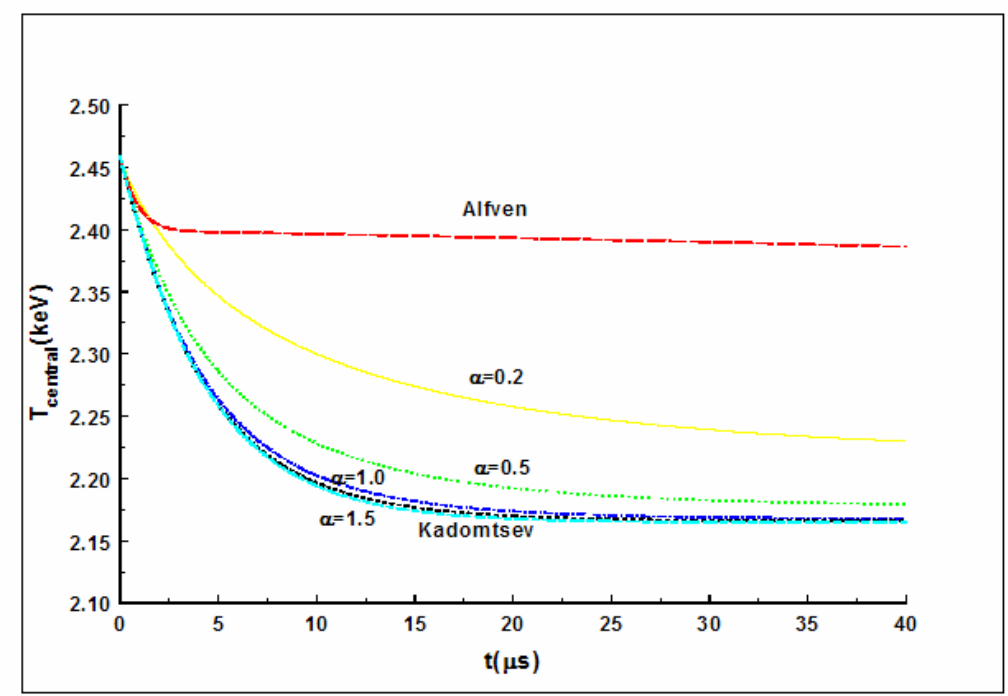

Figure 12. Temporal temperature evolution at the plasma center during the sawtooth crash.

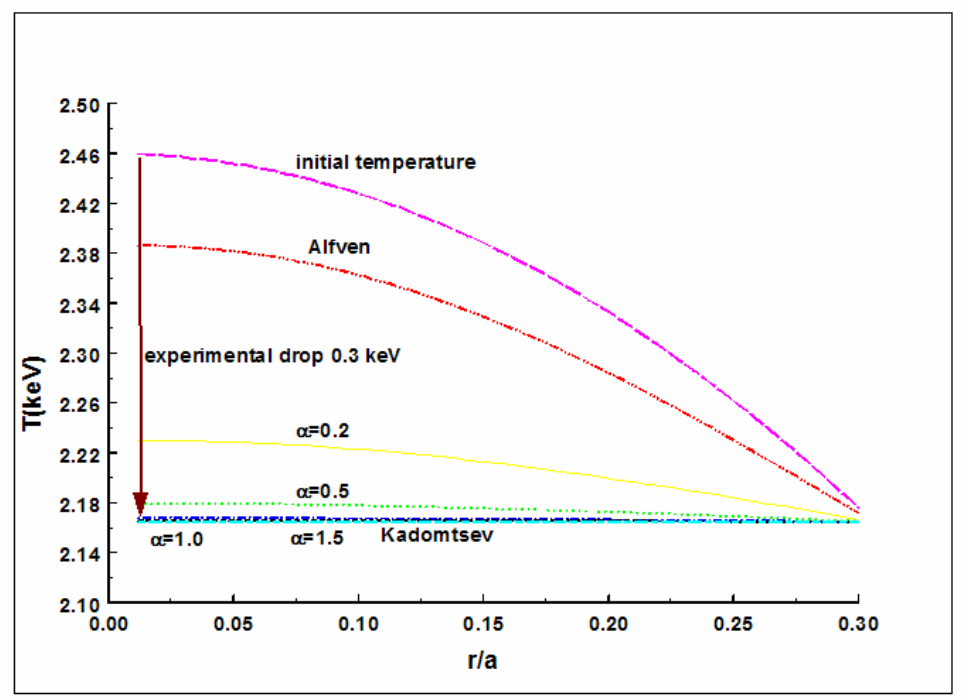

Figure 13. Temperature profile after $40 \mu s$. 
Comparing theoretical predictions shown in figures 10,12 and 13 with experimental data it is possible to determine the range for the parameter $\alpha$ which corresponds to the measured temperature flattening at the plasma core. Calculations with "slower" mode decay ( $\alpha=1-3)$ predict decrease of the central temperature of the order of $0.3 \mathrm{keV}$ during the crash time and flattening of the temperature profile at the plasma core which is in a very good agreement with ECE measurements. At the same time, significantly faster time scales like inverse Alfven time (ideal instability timescale) can not lower temperature in the plasma core. One can see in figure 13 that such a fast decay of instability is not sufficient to flatten the temperature profile inside the $q=1$ resonant surface during the crash time. Here the central decrease of the temperature is only 0.08 $\mathrm{keV}$ which is in clear contradiction with experimental values. Thus, we can conclude that the real damping is much slower than the inverse Alfven time, but of course it is much faster than the growth of the resistive $(1,1)$ mode before the sawtooth crash. This result suggests that evolution of the $(1,1)$ mode is strongly nonlinear not only before the crash, as shown in [4], but also during the crash time. It is important to emphasize that it is also difficult to determine the crash time because of the local nature of the sawtooth crash. The SXR signal modelling suggests that in the case considered the crash time is longer than $20 \mu s$ but shorter than $40 \mu s$. We have found that the obtained results hold also for the crash time $20 \mu \mathrm{s}$.

In our analysis we have used time-dependent and space-averaged diffusion coefficients. It is clear that in this way we can arrive at only general conclusions but not obtain detailed answers. From the mathematical point of view it is not a problem to solve the heat flow equation in the full 2D domain with local diffusion coefficients deduced by the mapping technique. The difficulty is related to the unknown boundary conditions which should be taken from experiment. This requires not only fast measurements, but also $2 \mathrm{D}$ measurements which have to be done in the sawtooth frame (co-rotation with the mode!). This, of course, is not feasible in real experiments. As a possible alternative to such measurements, one could think about reconstruction of a sawtooth crash based on at least a combination of 2D ECE and SXR measurements in reconstructing the temperature evolution. Solution of the $2 \mathrm{D}$ heat flow equation alone without such precise measurements and proper experimental boundary conditions can not improve the 
obtained results. This is the main reason why we operate with much more robust average values of the diffusion coefficient.

\section{Conclusions}

In this paper we have investigated two fast stochastic phenomena in fusion plasma in the ASDEX Upgrade tokamak: i) frequently interrupted regime (FIR-NTM) and ii) incomplete sawtooth reconnection. Using the experimentally measured perturbations and profiles we have deduced the heat diffusion coefficient by means of the mapping technique. It is found that the maximum values of the local field line diffusion coefficient are of the order $10^{-5}$ to $10^{-4} \mathrm{~m}^{2} / \mathrm{m}$ which corresponds to the electron thermal diffusivity of the order of $10^{2}$ to $10^{3} \mathrm{~m}^{2} / \mathrm{s}$. These diffusion coefficients were used as input into nonstationary diffusion equation allowing us to study time evolution of the two phenomena.

Calculations show that in the case of FIR-NTM the modelled time (tens of $\mu s$ ) needed for the temperature flattening is significantly shorter than the experimentally observed (hundreds of $\mu s$ ). This means that diffusion is not the limiting factor which determines the time scale of the temperature drop. The main constraint is related to the growth rate of the $(1,1)$ mode which couples the $(3,2)$ and $(4,3)$ modes. The temperature is redistributed during the very short time when the coupling is complete.

In the case of incomplete sawtooth reconnection, we observe that only a relatively small decay of the mode amplitude could explain the temperature flattening observed in the experiment. Fast time scales, for instance the inverse Alfven time, can not explain this phenomenon. This is a clear indication of nonlinear evolution of the $(1,1)$ mode not only before, but also during the crash itself.

The proposed method makes it possible by solving diffusion equation to connect diffusion coefficients with measured plasma perturbations. This approach allows us to obtain general information about fast stochastic phenomena in tokamaks. It requires very moderate computational efforts. It can be regarded as a phenomenological method of 
studying fast phenomena in fusion plasma which can be compared with purely theoretical extensive full nonlinear MHD calculations. Such nonlinear calculations were recently performed for the FIR-NTM phenomenon [12]. It is very encouraging and interesting that the results of these calculations reproduce the structure of this phenomenon almost identically as compared with the mapping technique used by us. However the resulting crash time is significantly larger than in our simulations, where it is of course taken from the experimental data.

Finally it should be mentioned that there are other methods used for the evaluation of magnetic field lines diffusion, in particular the so-called decorrelation trajectory method. In [13] this method was applied to calculate the diffusion coefficients in the slab geometry. It is interesting that for a relatively small magnetic turbulence (Kubo number $=0.1)$ and a shearless situation, the results obtained by the authors of [13] are similar with those obtained by us. The influence of the magnetic shear on the thermal diffusivity should be explored.

\section{References}

[1] Zohm H., Angioni C., Arslanbekov R. et al 2003 Nucl. Fusion 431570

[2] Dumbrajs O., Igochine V., Constantinescu D. and Zohm H. 2005 Phys. Plasmas 12 110704

[3] Igochine V., Dumbrajs O., Constantinescu D., Zohm H. and Zvejnieks G. 2006 Nucl. Fusion 46741

[4] Igochine V., Dumbrajs O., Zohm H. and Flaws A. 2007 Nucl. Fusion 4723

[5] Abdullaev S.S., Finken K.H. and Spatschek K.H. 1999 Phys. Plasmas 6153

[6] Abdullaev S.S., Finken K.H., Jakubowski M. and Lehnen M. 2006 Nucl. Fusion 46 S113

[7] Lichtenberg A.J., Itoh K., Itoh S.-I. and Fukuyama A. 1992 Nucl. Fusion 32495

[8] Biewer T.M. et al 2003 Phys. Rev. Lett. 91045004

[9] Tokar M.Z. wt al 2007 Plasma Phys. Control. Fusion 49395

[10] Günter S., Gude A., Marashek M., Sesnic S., Zohm H., ASDEX Upgrade team and Howell D. 2001 Phys. Rev. Lett. 87275001

[11] Park H.K., Mazzucato E., Luhmann N.C., Jr., Domier C.W., Xia Z., Munsat T., 
Donne A.J.H., Classen I.G.J., van de Pol M.J. and TEXTOR team 2006 Phys. Plasmas 1355907

[12] Lütjens H. and Luciani J.F. 2006 Phys. Plasmas 13112501

[13] Negrea M., Petrisor I. and Balescu R. 2004 Phys. Rev. E 70046409 